\section{Physics at HERA}

\section{THE PHYSICS CASE}

The proton comprises three main constituents, the so-called valance quarks, confined by extremely strong forces which can be regarded as being due to the exchange of force particles called gluons (both of these constituents are called partons). The gluons occasionally transform into quark-antiquark pairs which, like the gluons, are present in very large numbers. The interaction of an electron colliding with a proton at HERA energies allows the study at high resolution of crucial aspects of the proton's structure.
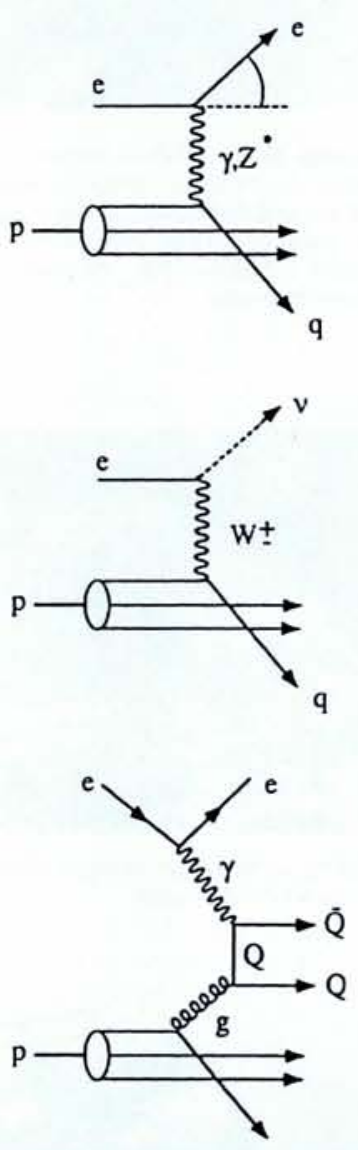

Fig. 1 - The basic processes contributing to deep-inelastic scattering: neutral current scattering (top), charged current scattering (middle), and photon-gluon fusion (bottom).

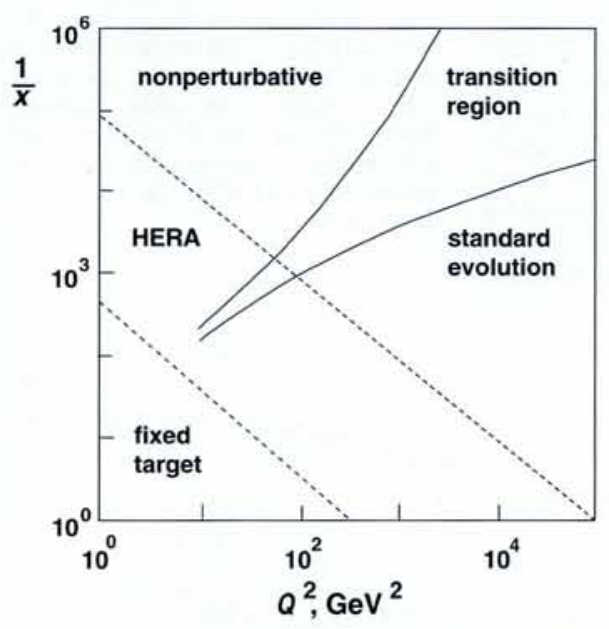

Fig. 2 - Kinematic region in $1 / x$ and $Q^{2}$ accessible with HERA and with a $600 \mathrm{GeV}$ muon beam incident on protons at rest.
Deep-inelastic lepton-nucleon scattering played a key rôle in the development of particle physics and in unraveling the internal structure of matter. From the early-1960s until today, experiments with electron, muon and neutrino beams at CERN, Cornell, DESY, Fermilab, and the Stanford Linear Accelerator Center running at centre-of-mass energies of 4 to $20 \mathrm{GeV}$ have led to major discoveries, such as the parton structure of the nucleon, the point-like nature of partons, their spin and fractional charge, the momentum distribution of partons in the nucleon, and through this the indirect discovery of the gluon content in the nucleon. The spin structure of the nucleon is also studied in deepinelastic scattering of polarized leptons off polarized nucleons. Last but not least, the weak neutral current was discovered in a deep-inelastic scattering experiment at CERN using a neutrino beam.

The structure of the proton is now being probed with an unprecedented accuracy using the electronproton storage ring HERA. The centre-of-mass energy in the collision of $30 \mathrm{GeV}$ electrons with $820 \mathrm{GeV}$ protons is $314 \mathrm{GeV}$, being a factor 15 larger than that of today's most energetic experiments. (To reach an energy of $300 \mathrm{GeV}$ in a fixed-target experiment one would need to accelerate leptons up to $50 \mathrm{TeV}$.) HERA's very high energy allows a completely new look at the inner structure of the proton since one will be able to resolve substructure at the scale of 1 part in 1000 of the size of the proton or better. HERA can thus be regarded as the world's most powerful microscope.

\section{Deep-Inelastic Scattering}

Deep-inelastic scattering is one of the three main physics issues accessible with HERA (others are photoproduction and the search for new forms of matter). In the simple quark-parton model of deep inelastic scattering, a quark is scattered out of the proton by the interaction with a virtual boson $\left(\gamma, Z^{0}\right.$ or $W^{-}$) emitted from the scattering lepton. Processes which proceed through the exchange of a neutral boson are called neutral current (NC) reactions, whereas the exchange of a charged boson is called a charged current (CC) reaction. Quantum chromodynamics (QCD) modifies this simple picture: gluons may be radiated before (initial-state radiation) or after (finalstate radiation) the boson-quark vertex, and the boson may also interact with a gluon inside the proton through an intermediate quark (boson-gluon fusion). The Feynman diagrams of Fig. 1 illustrate the basic processes which contribute to deep-inelastic scattering.

The deep-inelastic scattering process can be characterized by a set of kinematic variables, which in the case of a NC reaction are usually defined as follows:

$$
\begin{aligned}
& s=(k+p)^{2}=4 E_{\mathrm{e}} E_{\mathrm{p}} \\
& Q^{2}=-q^{2}=-\left(k-k^{\prime}\right)^{2} \\
& x=Q^{2} / 2 p q=Q^{2} / y s .
\end{aligned}
$$

Here, $k$ and $k^{\prime}\left(E_{\mathrm{e}}\right.$ and $\left.E_{\mathrm{e}^{\prime}}\right)$ are the four-momenta (energies) of the incoming and scattered electron, $p$ and $E_{\mathrm{p}}$ are the four-momentum and energy of the incoming proton, $x$, the Bjorken scaling variable, is the fraction of the nucleon momentum carried by the struck quark, and $Q^{2}$ is the square of the fourmomentum transferred by the exchanged current. Furthermore, $Q$ defines the resolution to which the photon probes the short-distance structure of the proton, i.e., the smallest object size $\Delta$ that can be resolved, $\Delta=\hbar / Q$.

The cross-section in deep-inelastic lepton scattering from an unpolarized nucleon is described in terms of structure functions which depend on $x$ and $Q^{2}$. To first order and for $Q^{2} \gg M_{Z}^{2}$, where $M_{Z}$ is the mass of the $Z^{0}$-boson, the cross-section for deep-inelastic electron scattering from an unpolarized proton can be expressed in terms of a structure function $F_{2}$ which depends on $x$ and $Q^{2}$ and the photoabsorption cross-section ratio of longitudinally and transversely polarized photons, $R=\sigma_{\mathrm{L}} / \sigma_{\mathrm{T}}$ :

$$
\frac{\mathrm{d}^{2} \sigma}{\mathrm{d} x \mathrm{~d} Q^{2}}=\frac{2 \pi \alpha^{2}}{x Q^{4}}\left(2[1-y]+\frac{y^{2}}{1+R}\right) F_{2}\left(x, Q^{2}\right)
$$

The structure function $F_{2}$ reflects the momentum distribution of the constituents of the nucleon. A measurement of the dependence of the structure function on $\mathrm{x}$ and $Q^{2}$ therefore provides information about the quark-distribution functions. $F_{2}$ has been measured in many previous experiments, and the most recent measurement from a fixed-target experiment covers $0.006<x<0.6$ and $Q^{2}$ up to $55 \mathrm{GeV}^{2}$.

With HERA, completely new domains in $x$ and $Q^{2}$ become accessible, as shown in Fig. 2. The very high energies accessible allow one to extend the range of four-momentum transfer $Q^{2}$ to almost $10^{5} \mathrm{GeV}^{2}$, corresponding to a spatial resolution of $10^{-18} \mathrm{~m}$. Of special interest at HERA is the physics at low $x\left(10^{-4}<x<10^{-2}\right)$ and high $Q^{2}\left(>1 \mathrm{GeV}^{2}\right)$, as it allows the test of QCD at very high parton densities. When looking at the constituents of a proton in the range $x>10^{-2}$ and moderate $Q^{2}$, one sees essentially the valence quarks which carry the major fraction of the proton momenta. According to the standard theory of QCD, one expects so-called evolution at very small $x$, i.e., the splitting of partons with a rapid increase in parton density. This increase diverges at some point so one expects that at very low $x$, the highly successful model of free, noninteracting partons fails and interactions between quarks and gluons become important. The measurement of $F_{2}$ at low $x$ thus allows a test of QCD in a transition region between perturbative and non-perturbative behaviour.

\section{Photoproduction}

The region of low $x$ and low $Q^{2}\left(Q^{2}<4 \mathrm{GeV}^{2}\right)$ is the kinematic regime where the photon emitted by the electron is no longer highly virtual, but quasireal. Therefore in HERA, not only electrons but also a "beam" of nearly real photons collides with the protons. The analysis of these photoproduction events provides information about the $\gamma p$-coupling and the hadronic content of the photon. 


\section{New Particles}

The search for new particles and phenomena is one of the central questions addressed with each new accelerator. In the standard model of electroweak and strong interactions, quarks and leptons, together with the gauge bosons, are the fundamental constituents of matter. Many extensions to the model have been proposed in attempts to understand unanswered questions (e.g., quark-lepton symmetry, the existence of three generations of fermions, the mass spectrum of these fermions). These extension, if true, should manifest themselves in phonomena which have not been detected so far. Coloured leptoquarks appear naturally in many theories that extend the symmetry of the standard model in order to unify the known forces, either in models postulating new interactions or in models assuming compositeness of quarks and gluons]. Excited states of known leptons are expected if the leptons are composite objects. If found, these states would represent a convincing proof of a new layer of structure in nature.

The electron-proton collider HERA is ideally suited to search for leptoquarks (massive coloured bosons with spin 0 or 1 , with lepton and baryon number and with fractional charge) and leptogluons (octet states with lepton numbers) since these particles could be produced as s-channel resonances in the electron-parton system, showing up as a resonance in the $x$-distribution of neutral current events at $x=M^{2} / s$, where $M$ is the leptoquark mass.

Searching for excited states of, for instance, electrons $\left(\mathrm{e}^{*}\right)$ and neutrinos $\left(v^{*}\right)$, is a natural way to investigate the possibilities of substructure. At HERA, excited electrons with masses up to the present limit of $296 \mathrm{GeV}$ would be produced directly by the process ep $\rightarrow \mathrm{e}^{*} \mathrm{X}$ (where $\mathrm{X}=\gamma, \mathrm{W}$ or $\mathrm{Z}$ ) allowing a search for $e^{*}$ using various decay modes.

\section{The $\mathrm{H} 1$ and ZEUS Experiments}

The main task of the HERA detectors is the accurate measurement of the kinematic quantities describing the ep-scattering process. In the case of a deep inelastic scattering process via the exchange of a neutral current, this corresponds to the measurement of four quantities: the energy and angle of the scattered electron, and the energy and angle of the struck quark. This puts very stringent requirements on the energy measurements for hadrons and electrons. The two experiments ( $\mathrm{H} 1$ and ZEUS) have thus paid considerable attention to the development of energy measurement using calorimeters, aiming at both an excellent absolute energy calibration and a good energy resolution. In doing so they have set new standards in calorimetry.

The key features of the detectors are:

- Lepton identification and measurement, i.e., electron identification, measurement of its energy and direction, e/p separation, and the identification of muons (resulting, e.g., from the decay of heavy quarks created in gluon-boson fusion).

- Measurement of the hadronic energy flow, requiring good jet-energy measurement, high granularity, hermeticity of the detector, and a highly stable absolute calibration.

- Tracking of charged particles in a magnetic field to measure the lepton charge, the momenta of the particles and to help with the topological analysis of the events, such as the jet analysis.

The luminosity is measured in both experiments using the rate of bremsstrahlung, ep $\rightarrow$ e'p $\gamma$, for which the cross-section is large and well-known. Both the photon and the scattered electron are detected in calorimeters located downstream of the interaction region in the direction the electrons move.

\section{FIRST RESULTS}

The results presented in this section are based on data collected in 1992 and 1993, corresponding to an integrated luminosity of data written on tape of about $600 \mathrm{nb}^{-1}$. Fig. 3 shows the development of the accumulated HERA luminosity as seen by the $\mathrm{H} 1$ luminosity monitor for the full data-taking period of 1993 . The figure underlines the very reliable operation of the machine and the steady increase in amount of accumulated data. Comparison with the data for 1992 demonstates an increase by a factor 20 of the integrated luminosity in 1993.

Electron-proton scattering is dominated by the exchange of almost real photons. While most of the photoproduction cross-section is due to soft processes, a fraction of the $\gamma p$-collisions at HERA energies are hard-scattering processes leading to high$p_{T}$ particles in the final state. Real photons can interact with the partons of the proton through two mechanisms. In the first case, the photon couples as a point-like gauge boson through the electromagnetic interaction directly to one of the partons of the proton, either to a quark (QCD Compton scattering) or to a gluon (boson-gluon fusion). This interaction is called a direct process. In the second case, the photon fluctuates into a vector meson which has the same quantum numbers as the photon and where the meson then interacts hadronically with the proton. This process has been known for a long time and is described by the vector dominance model (VDM). For large values of $p_{\mathrm{T}}$, the photon becomes the source of partons which interact with the partons of the proton. Those hadronic constituents of the photon not participating in the interaction with the proton give rise to a hadronic system, known as the

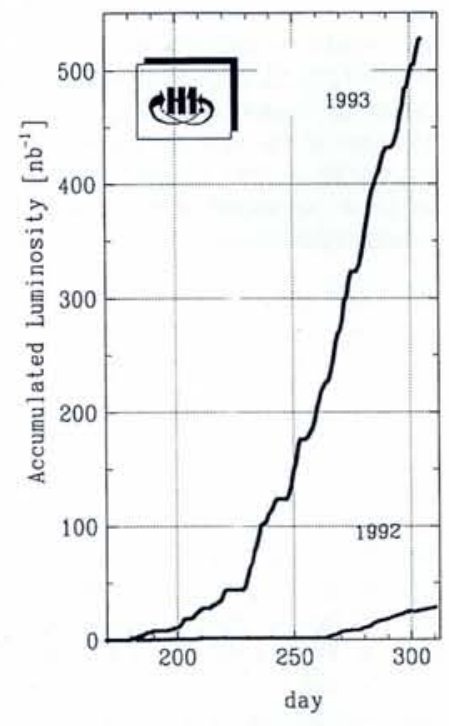

Fig. 3 - The increase with time of the accumulated luminosity (as seen by H1) during 1992 and 1993.

Further information about HERA physics and the first results can be found in Proc. Int. Europhysics Conf. on High Energy Physics (Marseilles, 22-28 Jully 1993). Eds.: J. Carr and M. Perrottet (Editions Frontières, 1994) pp. 791-824, and references therein.

\section{H1 Collaboration}

The $\mathrm{H} 1$ detector was built and is operated by a collaboration of 380 physicists from 35 institutions in 12 countries. Its main elements are a central and forward tracking system, including a transition radiation detector in the forward direction, inside a superconducting coil with a diameter of $6 \mathrm{~m}$ which provides a longitudinal field of 1.2 T. The scattered electron and the energy of the hadronic final state are monitored with a highly segmented, liquid argon calorimeter and a backward electromagnetic lead-scintillator calorimeter. The solenoid is surrounded by a segmented iron return yoke filled with limited streamer

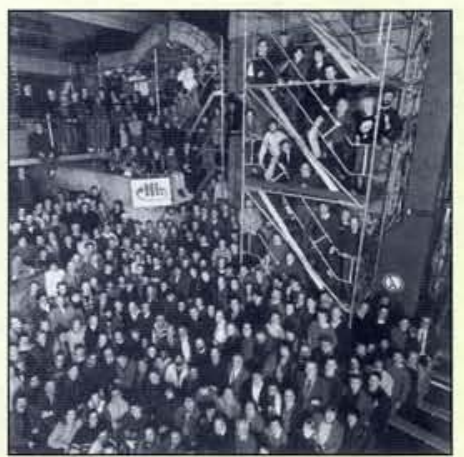

tubes to measure the hadronic energy leaking out of the calorimeter, and to identify and measure muon tracks. Muons are in addition measured by muon chambers placed inside and outside the yoke. In the forward region, a spectrometer with a toroidal magnet and drift chambers is used to measure forward-going high-momentum muons.

\section{ZEUS Collaboration}

The ZEUS detector was built and is operated by a collaboration of 430 physicists from 50 institutions in 12 countries. Charged particles are tracked using a system of chambers surrounded by a thin, superconducting solenoid providing a field of $1.43 \mathrm{~T}$. A uranium-scintillator calorimeter surrounds the tracking detectors and the solenoid. The calorimeter covers a solid angle equivalent to $99.7 \%$ of $4 \pi$, and sits inside a magnetized iron return yoke which is segmented and instrumented in the radial direction. It serves at the same time as a backing calorimeter to measure the energy leaking out of the uranium calorimeter

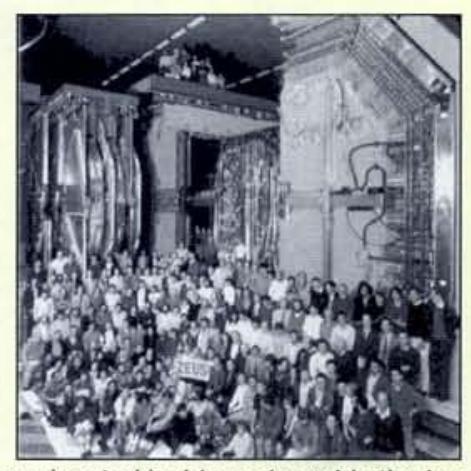
and as muon filter. Muons are measured in chambers located inside and outside the iron yoke. In the forward direction, high-momentum muons are measured in additional tracking chambers placed between iron toroids. 
Fig. 4 - The total photoproduction cross-section, measured at $\mathrm{H1}$ and ZEUS, together with data taken at lower energies, as a function of the $\gamma p$ centre-of-mass energy $W_{\gamma p}$. The dashed and solid lines represent different theoretical predictions.

Fig. 5 - The distribution of the fraction of the photon momentum carried by the interacting partons $x_{\gamma}$, as measured at ZEUS, for events with two or more jets. The data are plotted together with the calculated distributions for events with both resolved and direct processes (separate and combined).

Fig. 6 - An example of a CCevent as seen by the $\mathrm{H} 1$ detector. Shown are computer visualizations of the particle trajectories in the detector (lateral and end-on views) and a histogram of the energy flow as a function of the azimuthal and polar angles. The histogram shows the production of well-collimated hadrons from a current jet.
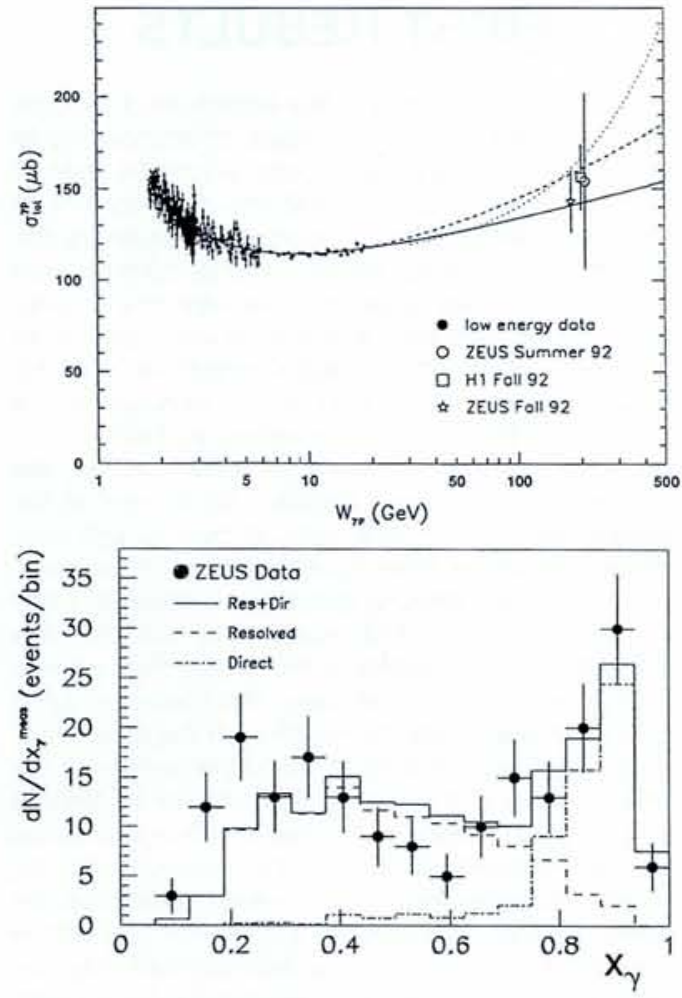

photon remnant, going approximately in the direction of the original photon. This type of interaction is called a resolved process. In another component of the resolved photoproduction, the photon splits in a quark-antiquark pair without forming a bound state. This part is called the anomalous or point-like component.

The $\nu$ p-interactions at HERA can reach centre-ofmass energies of $200 \mathrm{GeV}$, roughly one order of magnitude larger than the energies attained in fixedtarget experiments. This high energy gives access to a number of measurements hitherto considered impractical. The resolved and direct components of
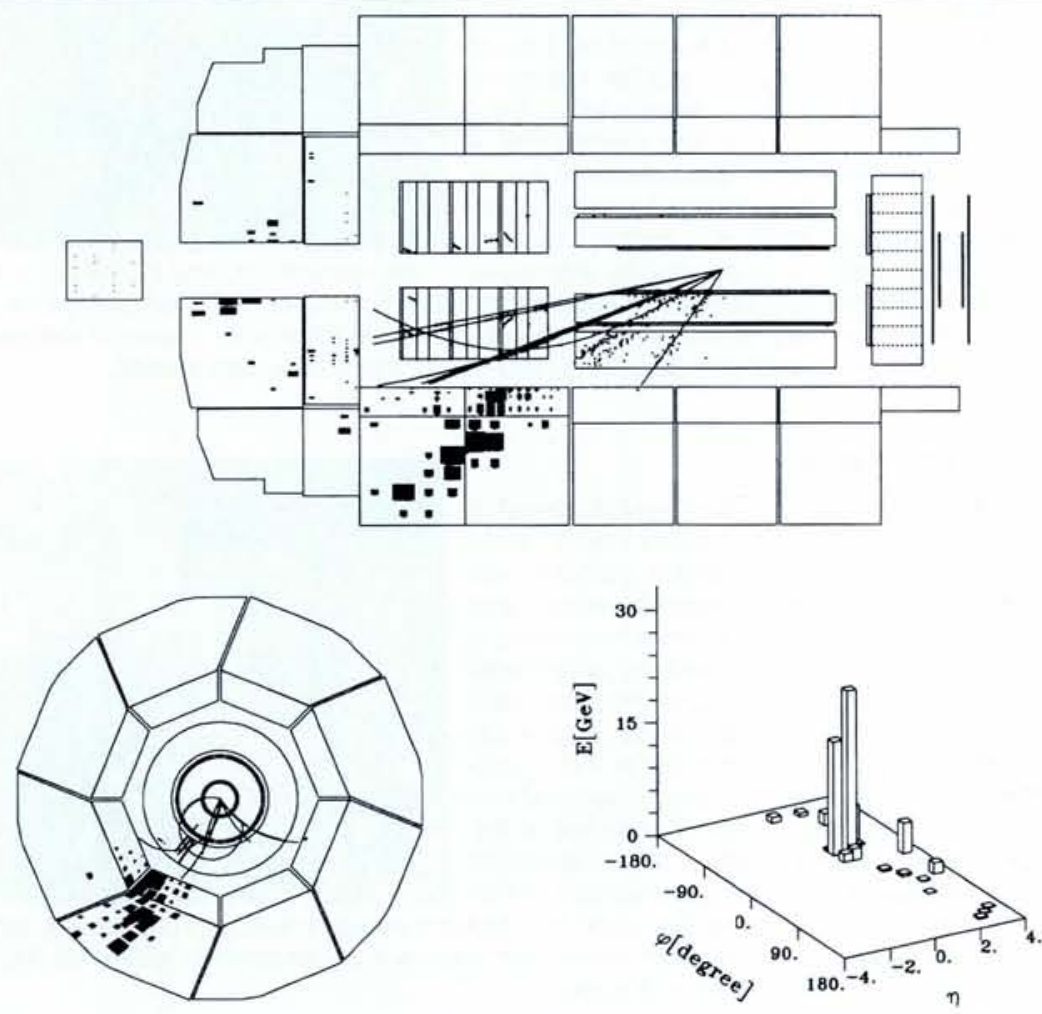

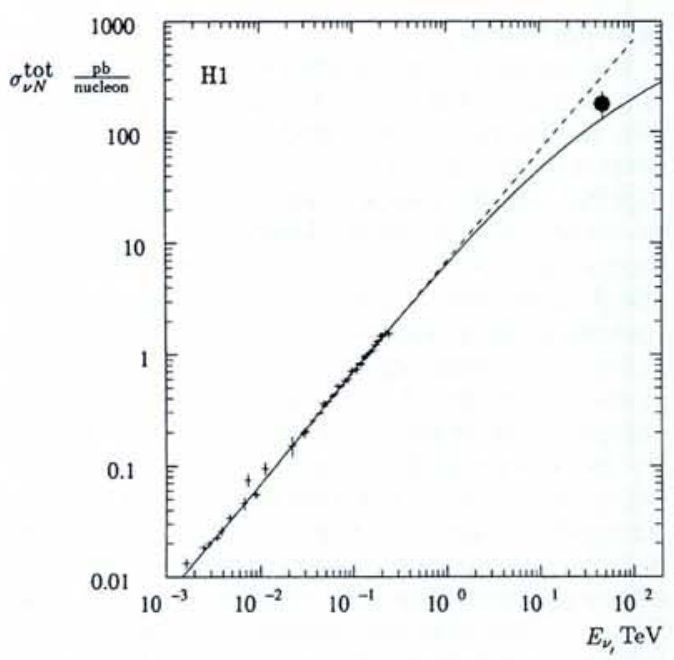

Fig. 7 - Dependence of the $\mathrm{N}$-cross-section on energy. The crosses indicate the data at low energies for neutrinos. The dot shows the results of the $\mathrm{H1}$ detector collaboration at DESY, transformed for comparison with the $\mathrm{N}$-cross-section and thereby corresponding to a measurement at $50 \mathrm{TeV}$. The solid line shows the cross-section calculated asuming the existance of a W-propagator and the dashed line represents a linear extrapolation of the data measured at lower energies.

the photon become distinguishable, making possible a measurement of the quark and gluon content of the photon.

\section{Photon-Proton Cross-Section}

Both HERA collaborations ( $\mathrm{H} 1$ and ZEUS) have published measurements of the total photon-proton cross-section at centre-of-mass energies of around $200 \mathrm{GeV}$. The results are shown in Fig. 4, corresponding to the following values: $\sigma_{T}(\gamma p)=156 \pm 2 \pm$ $18 \mu \mathrm{b}(\mathrm{H} 1)$ and $\sigma_{\mathrm{T}}(\gamma \mathrm{p})=133 \pm 3 \pm 16 \mu \mathrm{b}$ (ZEUS). Here, the first errors are the statistical errors and the second the systematic errors, which are mainly dominated by uncertainties in the detector acceptance. The results exclude models which predict a sharp rise of the cross-section (these models were motivated by measurements indicating an anomalously high muon content in cosmic-ray air showers). They also show that the cross-section rises only slowly with energy, as predicted by the so-called Regge model.

\section{Hard Photodiffraction}

Hard-scattering processes in photoproduction reveal the internal structure of the photon and allow the extraction of its parton densities. The signature is a final state with well-defined jets of hadrons. Both collaborations have successfully searched for jet structures in photoproduction events. A fraction of events shows a clear two-jet structure characteristic of the underlying hard, two-body, scattering process. From a measurement of the energies and angles of the jets in the two-jet sample one can estimate $x_{p}$ and $x_{\gamma}$, the fractions of momenta of the proton and the photon carried by the interacting partons. The measured distribution of $x_{\gamma}$ (shown in Fig. 5) rises both at low and high values of the centre-of-mass energy $W_{\gamma_{p}}$. The distributions expected for the resolved and direct processes are also shown in the figure. The majority of events originating from the resolved processes have an $x_{\gamma}$ less than 0.7 , whereas the contribution from the direct processes peaks near $x_{\gamma}=1$. The measured peak at high $x_{\gamma}$ therefore represents clear-cut evidence for direct processes. 

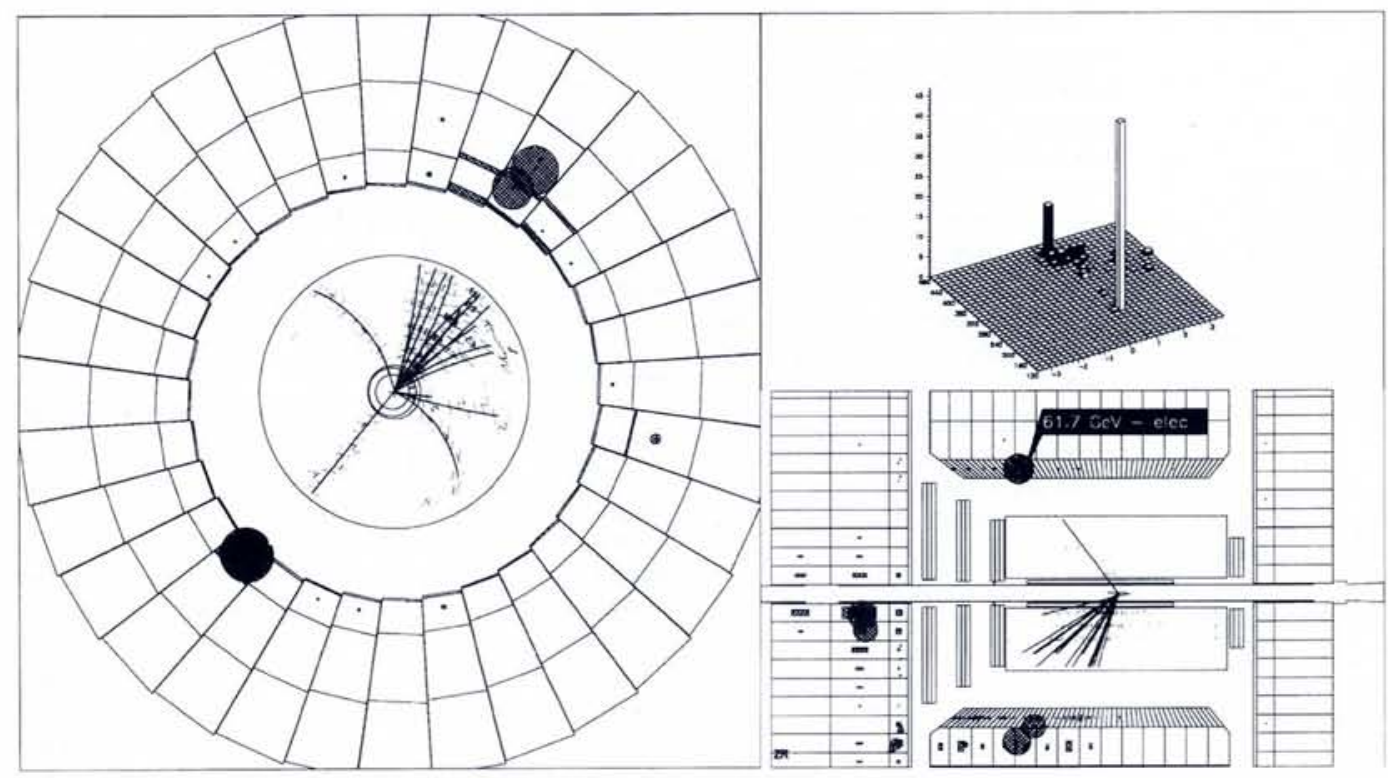

\section{Weak Interactions}

The deep-inelastic scattering process proceeds through either a neutral current (NC), i.e., the exchange of a photon (or a $Z^{0}$ at high $Q^{2}$ ), or a charged current (CC), i.e., the exchange of a W: As the differential cross-section for NC-events is proportional to $1 / Q^{4}$, most events registered at HERA so far cluster at moderate $Q^{2}$ and low $x$. Compared to previous experiments, HERA gives for the first time access to very low $x$-values in deep inelastic scattering with $Q^{2}>5 \mathrm{GeV}^{2}$.

The weak neutral current has been investigated using neutrino interactions for the last 30 years, starting with attempts to find the W-boson. Charged current events provide a tool to study weak interaction in a completely new kinematic regime, where the exchange of a $\mathrm{W}^{-}$plays a key rôle. Charged current events are characterized by a large momentum imbalance since the outgoing neutrino is not registered in the detector. This signature can be used to identify CC-events. Fig. 6 gives an example of a a CC-event where the well-collimated hadrons from the current jet are clearly visible in both lateral and end-on views. No visible particle in the opposite hemisphere balances the momentum, indicating the presence of a neutrino.

Using the data taken in 1993, both $\mathrm{H} 1$ and ZEUS have found events mediated by the exchange of a W-boson. Fig. 7 shows the cross-section for the process e $p \rightarrow v_{e}+$ hadrons, expressed in terms of the cross-section for $v \mathrm{~N}$-interactions, together with results from earlier experiments. The deviation from the linear increase in the cross-section with energy expected from the Fermi theory represents clear evidence for the existence of the W-propagator in the weak interaction. By comparing the relative rates of NC to CC events as function of $Q^{2}$, it is evident that the strengths of the electromagnetic and weak interactions become similar at $Q^{2}>10^{4} \mathrm{GeV}^{2}$.

\section{Proton Structure Function}

An example of a neutral current event with large $Q^{2}\left(Q^{2}=5400 \mathrm{GeV}^{2}\right)$ is shown in Fig. 8. It reveals the typical pattern of a NC-scattering process whereby the hadronic jet resulting from the struck quark is well balanced in momentum by the clearly identified, scattered electron in the other hemisphere. A certain amount of energy flow in the direction of flight of the proton (the forward direction) originates from the proton remnant. The study of NC-events has so far lead to a first measurement of the structure function $F^{2}$ and to the study of the hadronic final states. Analyses in both $\mathrm{H} 1$ and ZEUS proceed along similar lines, with the identification of clean, accurately measured events, the calculation of their kinematic variables, and the unfolding of the differential cross-sections for the effects of the detector resolution and radiative corrections.

The two experiments use somewhat different techniques in reconstructing the kinematics of the scattering process. In general, the kinematics are determined by two, independent, Lorentz-invariant variables. One normally uses $x, Q^{2}$ or $y$ which can be determined from the scattered electron variables (scattering angle and energy), from the hadron mixture of the two. Compared to previous experiments, the possibility of determining the kinematic variables in several ways provides an important redundancy as well as a cross-checking of systematic effects. The values for $F^{2}$ as function of $x$, as measured by both experiments, are shown in Fig. 9 . for four different values of $Q^{2}$ (similar curves are found for of $Q^{2}$ values in the range $8.5 \mathrm{GeV}^{2}<Q^{2}$ $<1000)$ Although statistical and systematic errors remain large, a number of conclusions can be drawn. notably that the results from the two experiments are in good agreement, and their normalization agrees with data obtained at lower energies. At low $x$, the structure function clearly increases with decreasing $x$. This is in contradiction with early Reggemodel predictions. The extrapolations and predictions of different parton distributions based on perturbative QCD are compared in Fig. 9 with the data. Several of these distributions describe the data quite well, whereas others tend to lie below the data.

\section{Colour Transfer}

In the quark-parton model, the hadronic final state in a deep-inelastic scattering electron-proton process consists of a jet of particles originating from the struck quark, the so-called current jet, and of particles originating from the remnant of the proton. The transverse momentum of the scattered electron (or neutrino) is balanced by the current jet, whereas the hadrons from the proton go forward, leaving the detector mostly unobserved (i.e., through the beam pipe) and carrying very little transverse momentum. This simple model is strongly modified by QCD through gluon radiation emitted owing to the colour transfer between the struck quark and the proton remnant. variables (jet energies and directions), or from a
Fig. 8 - An example of an NC event as seen by the ZEUS detector. Shown are computer visualizations of the particle trajectories (end-on and lateral views) and a histogram of the energy flow deposited as a function of the azimuthal and polar angles. Clearly resolved is a hadronic jet that is well balanced in momentum by a scattered electron in the ZEUS detector's other hemisphere.
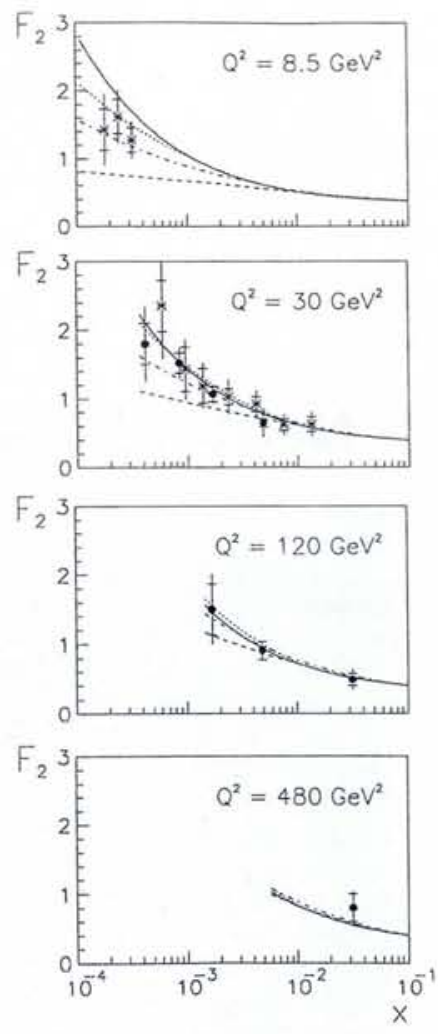

Fig. 9 - Measurement of the structure function $F_{2}$ as a function of $x$, for different values of $Q^{2}$ Data from $\mathrm{H1}$ (crosses) and ZEUS (dots) are compared with curves corresponding to theoretical predictions for various models. 


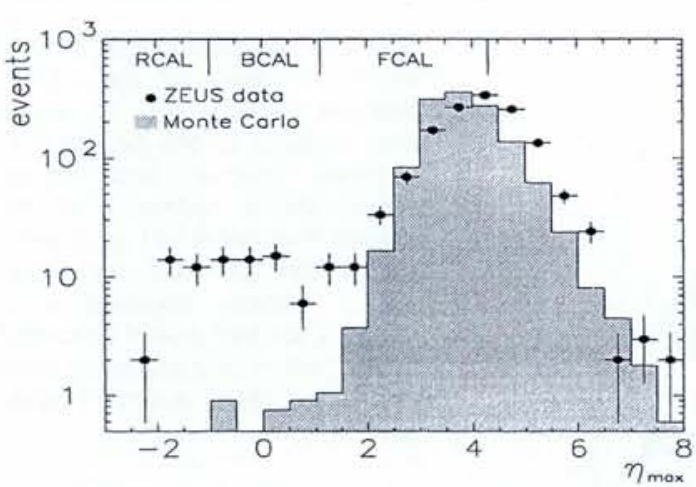

Fig. 10 - Distribution of $\eta_{\max }$, the maximum rapidity of a calorimeter cluster in an event. The data were obtained by the ZEUS Collaboration and the histogram shows the expectation for deepinelastic, neutral current events. Also indicated are the boundaries for the three calorimeter sections. pendence of $\alpha_{\mathrm{s}}$.
This transfer effect is clearly seen at HERA. Due to the large centre-of-mass energy, the jet structure resulting from the quarks and gluons emitted in the scattering process become clearly visible. Several jets can be observed in addition to the current jet and the jet resulting from the proton remnant. They arise owing to $Q C D$ processes such as gluon radiation in the initial and final states or photongluon fusion. Since the rate of three-jet events is proportional to the strength of the strong interaction $\left(\alpha_{\mathrm{s}}\right.$ being the strong-coupling constant), the $Q^{2}$ dependence of the three-jet rate can be used to study the $Q^{2}$ de-

The dominant mechanisms contributing to the cross-section of deep-inelastic scattering involve the interaction of the virtual photon emitted by the electron with a coloured parton originating from the proton. The resulting colour transfer between the struck quark and the proton remnant leads to a population of the interval between them with final-state hadrons. In the case of the HERA experiments, one would therefore expect a substantial energy flow at angles close to the proton beam direction. This is indeed observed for the large majority of events.

\section{Diffractive Interaction}

The ZEUS Collaboration has reported the first observation of a class of deep-inelatic scattering events in which no hadronic energy is deposited close to the proton beam direction. This observation was confirmed by the $\mathrm{H} 1$ Collaboration. The effect is best visible by studying the angular distribution of particles scattered into the detector, using a quantity called pseudorapidity $\eta$ related to the scattering angle $\theta$ whereby $\eta=-\ln (\tan \theta / 2)$. Fig. 10 shows the distribution of the largest scattering angle, $\eta_{\max }$, between the incoming proton and all outgoing particles. Also shown, as a shaded histogram, is the distribution of $\eta_{\max }$ as expected from a simulation similar to that used in the analysis of $F_{2}$ (see above), where the results of the simulation are insensitive to the exact choice of the model parameters. A comparison between the data and the simulation reveals an obvious excess of events with a "large rapidity gap" that is not described by the standard QCD-inspired models of fragmentation. The general features of these events, such as the flat rapidity distribution and the distribution of the invariant mass of the observed hadronic system, suggest a diffractive interaction between the photon and the proton, through the exchange of a colourless object.

\section{New Particles}

Coloured leptoquarks appear naturally in many theories beyond the Standard Model. The ep-collider HERA is an ideal machine to look for leptoquarks and leptogluons that could be produced as s-channel resonances in the electron-parton system. Excited states of known leptons would, if found, constitute strong proof for the existence of a substructure of the known leptons. Neither $\mathrm{H} 1$ nor ZEUS has observed a signal in the $x$-distribution. Each has derived mass limits on leptoquarks as a function of the left- and right-handed coupling strength at the electron-quark-leptoquark vertex. The $\mathrm{H} 1$ Collaboration has also looked for leptogluons but has found no evidence. The search for excited leptons $\left(e^{*}\right.$ and $\left.v^{*}\right)$ included an investigation of several decay topologies. Although no evidence has been found for the production of $e^{*}$ and $v^{*}$, limits on the coupling strength of an excited lepton have been determined as function of mass.

\section{Industrietagung Synchrotronstrahlung} 15./16. September 1994

Informationstage über die industrielle Nutzung von Synchrotronstrahlung beim Forschungszentrum DESY in Hamburg. Seit 1993 wird der Speicherring DORIS ausschliesslich als Quelle für Synchrotronstrahlung - speziell im Röntgenbereichen - betrieben. DESY kann deshalb sein Angebot zur Nutzung dieser hochwertigen Strahlung für Forschungs- und Entwicklungsarbeiten wertigen Strahlung tur Forschungs- und Entwicklungsarbeiten keiten und das Polential anwendungsbezogener Forschung zum Beispiel aut den Gebieten der Obentachenphysik, Molekularbiologie, Katalyse, Material- und Geowissenschaften. Die Tagung wendet sich insbesondere auch an kleine und mittelständische Industrieunternehmen

Weitere Informationen/Anmeldung:

DESY - HASYLAB-Industrietage

Notkestrasse 85,22603 Hambur

Tel. (040) 8998-4600 Fax (040) 8998-2787

\section{Neutrino Astrophysics}

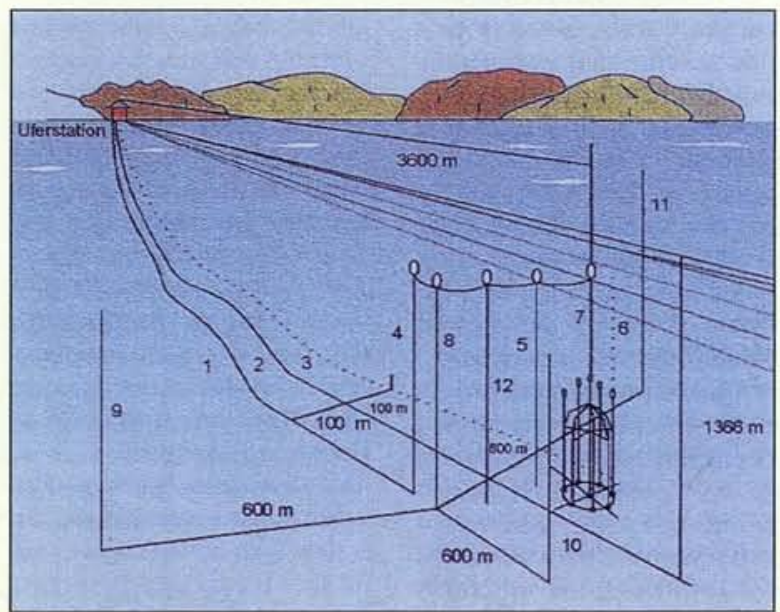

A schematic illustration of the NT-200 pilot neutrino telescope experiment in Lake Baikal. The detector modules are suspended some $1 \mathrm{~km}$ underwater in the two-tier cage-like array shown on the right. The various lines shown in the figure are for mooring and communications.
A device to detect neutrinos in the TeV energy range from high-energy cosmic sources is being actively pursued by DESY-Zeuthen in cooperation with Russian groups. In April 1993, the collaboration succeeded in deploying a portion of a future underwater neutrino telescope. This pilot array consists of 36 photomultipliers arranged in a three-dimensional lattice at a depth of more than $1 \mathrm{~km}$. The approximate energy and direction of muons transversing the water near the detector modules is determined by observing the Cerenkov light emitted by the high-energy muons as they interact with the water. The array is the first of its kind to operate, and although by itself it is too small to search for cosmic sources of neutrinos, it serves as a system to test underwater technologies and procedures for interpreting the data and optimizing detector parameters significant steps towards building a neutrino telescope. Data obtained so far lead to the conclusion that an arrangement consisting of 200 or more photomultipliers in Lake Baikal would offer a realistic chance to detect neutrinos from cosmic sources. The pilot array is also used to search for magnetic monopoles, and upper limits for their fluxes can be set.

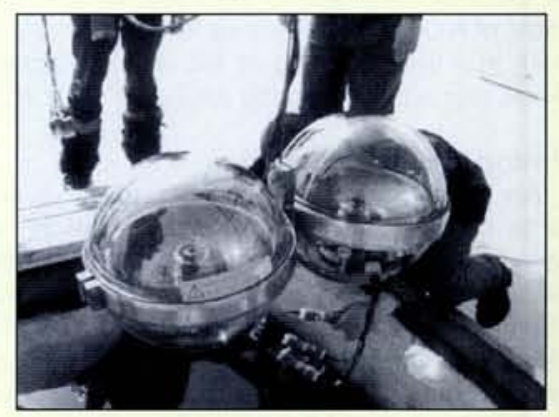

Deploying a pair of detector modules in Lake Baikal 